\title{
Dynamic Systems with Baseline Exponential Distribution Based on Sequential Order Statistics Under a Power Trend for Hazard Rates
}

\author{
Majid Hashempour ${ }^{1, \star}$, Mahdi Doostparast ${ }^{2}$ \\ ${ }^{1}$ Department of Statistics, School of Sciences, University of Hormozgan, Bandar Abbas, Iran \\ ${ }^{2}$ Department of Statistics, School of Mathematical Sciences, Ferdowsi University of Mashhad, Mashhad, Iran
}

\section{ARTICLE INFO}

Article History

Received 06 Jan 2018

Accepted 19 Aug 2019

Keywords

Proportional hazard rates

Hypotheses testing

Sequential order statistics

Estimation

Mathematics Subject

Classification: 62P30; 62N05;

$62 \mathrm{G} 30$.

\section{ABSTRACT}

This paper deals with analyzing dynamic engineering systems consisting of independent components. The failure of a components causes more load on the surviving components. This property is modeled by a power trend conditionally proportional hazard rates. For modeling system lifetimes, the theory of sequential order statistics can be used. Sequential order statistics coming from heterogeneous exponential distributions are considered. The maximum likelihood and Bayesian estimates of the parameters are obtained in different cases. The generalized likelihood ratio and the Bayesian tests are also derived for testing homogeneity of the baseline exponential component lifetimes arising from $s$ independent engineering systems.

() 2020 The Authors. Published by Atlantis Press SARL. This is an open access article distributed under the CC BY-NC 4.0 license (http://creativecommons.org/licenses/by-nc/4.0/).

\section{INTRODUCTION}

Let $X_{1}, \cdots, X_{n}$ be independent and identically distributed (i.i.d.) random variables with a common distribution function (DF), say $F$, and abbreviated by $X_{1}, \cdots, X_{n} \stackrel{\text { i.i.d. }}{\sim} F$. Denote in magnitude order of $X_{1}, \cdots, X_{n}$ by $X_{1: n} \leq \cdots \leq X_{n: n}$, called order statistics (OSs). The theory of OSs has been widely assumption in literature. For example, in system reliability analyses, lifetimes of $r$-out-of- $n$ systems coincide to $X_{r: n}$ where $X_{1}, \cdots, X_{n}$ stand for component lifetimes; For more information, see Barlow and Proschan [1] and David and Nagaraja [2] and references therein.

In this setting, failure of a component does not effect the surviving components. There are various practical situations in which this investigated and applied does not hold. For illustration, suppose that there exist $n$ power generators with nominal capacity $G_{1}, \cdots, G_{n}$. The system perform satisfactory if the total generated power is at least a given threshold, say $A$. If a generator fails, then the remaining generators have to generate much powers to fulfill the nominal level $A$ and hence more loads cause more pressure on the working generators. Therefore, the generator lifetimes are decreased. For statistical modeling these kinds of systems, some generalizations of OSs such as fractional OSs and generalized OSs have been introduced in literature. They are useful for providing more flexible tools and also a setting to unify similar results (David and Nagaraja [2], p. 21). In this paper, we deal with another unified concept, called the sequential order statistics (SOS), which has also a motivation in reliability analyses as discussed above. Specifically, when the component lifetimes are i.i.d., the OSs are suitable for describing the $r$-out-of- $n$ system lifetime. Here failing a component does not effect the DFs of lifetimes of surviving components. Motivated by Cramer and Kamps [3], the failure of a component may result in a higher load on the surviving components and hence causes the lifetime distributions change. More precisely, suppose that $F_{j}$, for $j=1, \cdots n$, denotes the common DF of the component lifetimes when $n-j+1$ components are jointly working. Then, the components begin to work independently at time $t=0$ with the common DF $F_{1}$. If at time $x_{1}$, the first component failure occurs, then the remaining $n-1$ components are working with the (left truncated) common DF $F_{2}$ at $x_{1}$. This process continues up to $n-r+1(r=1, \cdots, n)$ components with the common DF $F_{r}$ work until the $r$-th failure occurs at time $x_{r}$ and hence the whole system fails. The mentioned system is called sequential $r$-out-of- $n$ system or dynamic system and the system lifetime is then $r$-th observed component failure time, denoted by $X_{(r)}^{\star}$. In the literature, $\left(X_{(1)}^{\star}, \cdots, X_{(n)}^{\star}\right)$ is called SOSs. Statistical properties of SOSs have been studied by Kamps [4,5], Cramer and Kamps [3,6], Balakrishnan et al. [7], Beutner and Kamps [8], Hashempour [9], Bedbur

${ }^{\star}$ Corresponding author. Email: ma.hashempour@hormozgan.ac.ir 
[10], Hashempour and Doostparast [11], and references therein. We considered the problem of estimating the parameters on the basis of $s(\geq 2)$ independent SOSs samples under a new proposed power trend conditional proportional hazard rates (PTCPHR) model, defined by $F_{j}(t)=1-\left(1-F_{0}(t)\right)^{a^{j}}$ for $j=1, \cdots, r$ and $a>0$, where the underlying CDF $F_{0}(t)$ is assumed to be the exponential distribution, i.e.,

$$
F_{0}(x ; \sigma)=1-\exp \left\{-\left(\frac{x}{\sigma}\right)\right\}, x>0, \sigma>0
$$

In this case, the hazard rate function of the $\operatorname{CDF} F_{j}$, defined by $h_{j}(t)=f_{j}(t) / \bar{F}_{j}(t)$ for $t>0$ and $j=1, \cdots, n$, is proportional to the hazard rate function of the baseline DF $F_{0}$, i.e., $h_{j}(t)=a j h_{0}(t)$ for $t>0$.

In this paper, we consider the problem of comparing non-homogeneous exponential populations on the basis of independent multiply SOS samples coming from non-homogeneous exponential populations under the abovementioned PTCPHR model. Thus, this paper is organized as follows: In Section 2, via the likelihood approach, statistical procedures including estimation, either point or interval, of the parameters as well as the problem of testing homogeneity of baseline exponential populations are considered. In Section 3, Bayesian approach is used for estimating parameters of interest. Finally, concluding remarks are given in Section 4.

\section{LIKELIHOOD ANALYSIS}

In the sequel, suppose that we have observed $s(\geq 2)$ independent heterogeneous SOS samples. The available data may be represented as

$$
\mathbf{x}=\left[\left[x_{i j}\right]\right]_{i=1, \cdots, s, j=1, \cdots, r}
$$

where the $i$-th row of the matrix $\mathbf{x}$ in (2) denotes the SOS sample coming from the $i$-th population. The likelihood function (LF) for a single SOS sample is

$$
L\left(F_{1}, \cdots, F_{r} ; \mathbf{x}\right)=\frac{\Gamma(n+1)}{\Gamma(n-r+1)}\left\{\prod_{j=1}^{r-1} f_{j}\left(x_{j}\right)\left(\frac{\bar{F}_{j}\left(x_{j}\right)}{\bar{F}_{j+1}\left(x_{j}\right)}\right)^{n-j}\right\} f_{r}\left(x_{r}\right) \bar{F}_{r}\left(x_{r}\right)^{n-r}
$$

Therefore, the LF of the available data given by (2) is then obtained from (3) as

$$
L(\mathcal{F} ; \mathbf{x})=\left(\frac{n !}{(n-r) !}\right) \prod_{i=1}^{s}\left(\prod_{j=1}^{r-1}\left[f_{j}^{[i]}\left(x_{i j}\right)\left(\frac{\bar{F}_{j}^{[i]}\left(x_{i j}\right)}{\bar{F}_{j+1}^{[i]}\left(x_{i j}\right)}\right)^{n-j}\right] f_{r}^{[i]}\left(x_{i r}\right) \bar{F}_{r}^{[i]}\left(x_{i r}\right)^{n-r}\right),
$$

where $\mathcal{F}=\left\{F_{j}^{[i]}, i=1, \cdots, s, j=1, \cdots, r\right\}$ and for $i=1, \cdots, s, j=1, \cdots, r, \bar{F}_{j}^{[i]}(x)=1-F_{j}^{[i]}(x)$, and $F_{j}^{[i]}$ calls for the common DF of component lifetimes of the $i-$ th dynamic system. For more details, see Cramer and Kamps [6,12] and Hashempour and Doostparast [11]. Suppose that, the baseline DF of the $i$-th population $(i=1, \cdots, s)$ follows the one-parameter exponential distribution with the mean $\sigma_{i}$. Substituting Equation (1) into Equation (4) and under the earlier mentioned PTCPHR model in Section 1, the LF of the available data reads

$$
\begin{aligned}
L\left(\sigma_{1}, \cdots, \sigma_{s}, a ; \mathbf{x}\right) & =B^{s}\left(\prod_{j=1}^{r} a^{j}\right)^{s} \prod_{i=1}^{s}\left(\frac{1}{\sigma_{i}}\right)^{r} \prod_{i=1}^{s} \prod_{j=1}^{r} \exp \left\{-\frac{x_{i j} m_{j}}{\sigma_{i}}\right\} \\
& =B^{s}\left(a^{\frac{s r(r+1)}{2}}\right)\left(\prod_{i=1}^{s} \sigma_{i}\right)^{-r} \exp \left\{-\sum_{i=1}^{s} \sum_{j=1}^{r}\left(\frac{x_{i j} m_{j}}{\sigma_{i}}\right)\right\},
\end{aligned}
$$

where $B=(n ! /(n-r) !)$ and $m_{j}=(n-j+1) a^{j}-(n-j) a^{j+1}$ with convention $(n-r) a^{r+1} \equiv 0$. For sake of brevity, we assumed that the proportional parameter $a$ are the same among the $s$ sequential $r$-out-of- $n$ systems. In this section, we consider the problem of homogeneity testing on the basis of independent SOS samples from different exponential populations, i.e.,

$$
\left\{\begin{array}{l}
H_{0}: \sigma_{1}=\cdots=\sigma_{s} \\
H_{1}: \sigma_{i} \neq \sigma_{j} \exists i \neq j
\end{array}\right.
$$

In sequel, two cases are considered: (i) $a$ known, and (ii) $a$ unknown.

If $a$ is known, the results of Cramer and Kamps ([6], Chapters 4 and 5) can be used. Also, see Schenk [13]. A summarize for this case is given in the appendix A. 
Suppose that the parameter $a$ in Equation (5) is unknown. In this case, calculations are complicated. Here, the Hessian matrix (H) is defined by

$$
\mathbf{H}=\left[\left[\left(\partial^{2} \log (L) / \partial \theta_{i} \partial \theta_{j}\right)_{1 \leq i, j \leq s+1}\right]\right]
$$

where $\theta_{i}=\sigma_{i}(1 \leq i \leq s)$ and $\theta_{s+1}=a$. After some algebraic calculations, the Hessian matrix simplifies to

$$
\mathbf{H}=\left(\begin{array}{ll}
\mathbf{B}_{11} & \mathbf{B}_{12} \\
\mathbf{B}_{21} & \mathbf{B}_{22}
\end{array}\right)
$$

where

$$
\begin{aligned}
& \mathbf{B}_{11}=\operatorname{diag}\left\{\frac{r}{\sigma_{i}^{2}}-\frac{2 \sum_{k=1}^{r} a^{k}(n-k+1) D_{i j}}{\sigma_{i}^{3}}\right\}_{i=1, \cdots, s}, \\
& \mathbf{B}_{22}=\operatorname{diag}\left\{\frac{-s r}{a^{2 j}}\right\}_{j=1, \cdots, r}, \\
& \mathbf{B}_{\mathbf{1 2}}=\mathbf{B}_{21}^{\mathbf{T}}=\left[\left[\frac{(n-j+1) D_{i j}}{\sigma_{i}^{2}}\right]\right]_{i=1, \cdots, s, j=1, \cdots, r},
\end{aligned}
$$

where $\operatorname{diag}\left\{c_{i}\right\}_{i=1, \cdots, m}$ stands for a diagonal matrix with elements $c_{1}, \cdots, c_{m}$ on its main diagonal.

The Hessian matrix (7) is not necessary negative definite on the parameter space. Therefore, one needs to use numerically methods for maximizing the $\operatorname{LF}(5)$ with respect to $a$ and $\sigma_{1}, \cdots, \sigma_{s}$. Note that the ML estimates of the parameters (if exist) are obtained numerically by solving the following likelihood equations:

$$
\left\{\begin{array}{l}
\hat{\sigma}_{i}=\frac{1}{r} \sum_{j=1}^{r} x_{i j} \hat{m}_{j}=\frac{\hat{a}}{r} \sum_{j=1}^{r}(n-j+1) j D_{i j}, i=1, \cdots, s, \\
\hat{a}=r s\left(\sum_{i=1}^{s} \sum_{j=1}^{r}(n-j+1) j D_{i j} / \hat{\sigma}_{i}\right)^{-1} .
\end{array}\right.
$$

Here another approach for deriving the ML estimates of the parameters is suggested which is numerically simple. Recall that the profile LF for the parameter $a$ is derived by substituting $\hat{\sigma}_{i}$ given by (29), for $i=1, \cdots, s$, into the $\operatorname{LF}(5)$ instead of $\sigma_{i}$, i.e., $L_{p}(a ; \mathbf{x})=L\left(\hat{\sigma}_{1}, \cdots, \hat{\sigma}_{s}, a ; \mathbf{x}\right)$. The logarithm of the profile LF is

$$
\begin{aligned}
l_{p}(a ; \mathbf{x}) & =\log L_{p}(a ; \mathbf{x}) \\
& =\frac{s r(r+1)}{2} \log (a)-r \sum_{i=1}^{s} \log \left(\frac{\sum_{j=1}^{r}(n-j+1) a^{j} D_{i j}}{r}\right)-r s .
\end{aligned}
$$

One may maximize the function (8) with respect to the parameter $a$ numerically to derive the ML estimate of the parameter $a$. Then substituting this estimate into (29) (the appendix), the ML estimate of $\hat{\sigma}_{j}$, for $i=1, \cdots, s$, is derived.

Consider again the hypotheses testing problem (6). It is easy to verify that the unique ML estimates of the parameters under the null hypothesis $H_{0}$ are given by

$$
\hat{\sigma}_{0}=\frac{1}{r s} \sum_{i=1}^{s} \sum_{j=1}^{r} x_{i j} \hat{m}_{0, j}=\frac{\hat{a}_{0}}{r s} \sum_{i=1}^{s} \sum_{j=1}^{r}(n-j+1) j D_{i j}
$$

and

$$
\hat{a}_{0}=\frac{r s}{\sum_{i=1}^{s} \sum_{j=1}^{r}(n-j+1) j D_{i j}} \hat{\sigma}_{0},
$$

where $\hat{m}_{0, j}=(n-j+1) j \hat{a}_{0}-(n-j)(j+1) \hat{a}_{0}$, with convention $\hat{a}_{0}(r+1) \equiv 0$. Therefore, the generalized likelihood ratio test $($ GLRT $)$ statistic for the hypotheses testing problem (6) is

$$
\Lambda_{2}=\prod_{j=1}^{r}\left(\frac{\hat{a}_{0}}{\hat{a}}\right)^{r s} \prod_{i=1}^{s}\left(\frac{\hat{\sigma}_{i}}{\hat{\sigma}_{0}}\right)^{r} \exp \left\{\sum_{i=1}^{s} \sum_{j=1}^{r}\left(\frac{\hat{m}_{j}}{\hat{\sigma}_{i}}-\frac{\hat{m}_{0, j}}{\hat{\sigma}_{0}}\right) x_{i j}\right\},
$$

where $\hat{m}_{j}=(n-j+1) \hat{\alpha}_{j}-(n-j) \hat{\alpha}_{j+1}$. The logarithm of the GLRT statistic $\Lambda_{2}$ in Equation (11) reads

$$
\log \Lambda_{2}=r s \log \left(\frac{\hat{a}_{0}}{\hat{a}}\right)+r \sum_{i=1}^{s} \log \left(\frac{\hat{\sigma}_{i}}{\hat{\sigma}_{0}}\right)+\sum_{i=1}^{s} \sum_{j=1}^{r}\left(\frac{\hat{m}_{j}}{\hat{\sigma}_{i}}-\frac{\hat{m}_{0, j}}{\hat{\sigma}_{0}}\right) x_{i j} .
$$


The null hypothesis $H_{0}$ rejects if

$$
-2 \log \Lambda_{2}>c
$$

where $c$ is a positive constant and determined on the basis of the test level (Lehmann and Romano [14]).

Exact distribution of the statistic $-2 \log \Lambda_{2}$ in Equation (13) under the null hypothesis $H_{0}$ is complicated and we could not obtained an explicit expression. This remains as an open problem. In practice, one may use numerical methods such as Monte Carlo simulation study to derive the threshold $c$ in the rejection region (13).

\section{SOS-BASED BAYES ANALYSIS}

Bayesian statistical inference on the basis of homogeneous multiply SOS samples have been considered in literature. For example, Mohie El-Din et al. [15] considered the problem of SOS-based Bayesian estimation and one-sample prediction under the CPHR model for Weibull and Pareto distributions. Schenk et al. [16] derived Bayes estimates of the parameters under the CPHR model when the baseline population follows the one- and two-parameter exponential distributions. Also, the Bayesian estimation and the two-sample Bayesian prediction based on homogeneous multiply SOS samples was studied with more details by Shafay et al. [17]. See also Hashempour and Doostparast [11]. In this section, we consider the problems of estimation, either point or interval, of parameters and hypotheses testing (6) on the basis of $s$ independent heterogeneous SOS samples arising from the exponential populations under the PTCPHR model with known $a$. When the parameter $a$ is unknown, the mentioned problems are complicated and works in this direction are under investigation and we hope to report findings in future.

\subsection{Bayesian Point Estimates}

We here consider the problem of estimating unknown parameters via a strict Bayesian approach. To do this, we assume that $a$ is known and suggest the conjugate prior distributions for the scale parameters $\sigma_{i}, i=1, \cdots, s$, i.e.,

$$
\sigma_{i} \sim \operatorname{IG}\left(d_{i}, c_{i}\right), i=1, \cdots, s,
$$

be independent random variables where $I G(d, c)$ calls for the inverse gamma distribution with parameters $d$ and $c$ and density function

$$
\pi(\sigma)=\frac{c^{d}}{\Gamma(d)} \sigma^{-(d+1)} \exp \left\{-\left(\frac{c}{\sigma}\right)\right\}, \sigma>0, d>0, c>0
$$

From Equation (14) and the $\operatorname{LF}(5)$, the joint posterior density function of $\sigma_{1}, \ldots, \sigma_{s}$ is readily obtained as

$$
\begin{aligned}
\pi\left(\sigma_{1}, \ldots, \sigma_{s} \mid \underline{\mathbf{x}}\right) \equiv & \prod_{i=1}^{s}\left(B^{s} a \frac{r(r+1)}{2} \frac{c_{i}^{d_{i}}}{\Gamma\left(d_{i}\right)} \sigma_{i}^{-\left(d_{i}+r\right)-1}\right) \\
& \times \prod_{i=1}^{s}\left(\exp \left\{-\left(\frac{\sum_{j=1}^{r}(n-j+1) a^{j} D_{i j}+c_{i}}{\sigma_{i}}\right)\right\}\right),
\end{aligned}
$$

which implies

$$
\sigma_{i} \mid \underline{\mathbf{x}} \sim I G\left(d_{i}+r, \sum_{j=1}^{r}(n-j+1) a^{j} D_{i j}+c_{i}\right), i=1, \cdots, s .
$$

As we expected given $\underline{\mathbf{x}}$, the parameter $\sigma_{i}$ are independent. Under the squared error loss function, the Bayes estimate of the parameter is the mean of the associate posterior distribution. Here, the Bayes estimate of $\sigma_{i}$ is

$$
\hat{\sigma}_{i, B}=\frac{\sum_{j=1}^{r}(n-j+1) a^{j} D_{i j}+c_{i}}{d_{i}+r-1}=\frac{r \hat{\sigma}_{i}+c_{i}}{d_{i}+r-1}, \quad i=1, \cdots, s,
$$

where $\hat{\sigma}_{i}$ is the ML estimate of $\sigma_{i}$ given by Equation (29). Since the SOS samples are independent, the Bayes estimates in Equation (18) depend only on the respective samples. 
Remark 3.1. For $i=1, \cdots, s$ :

- The mode of the posterior distribution (17) is

$$
\hat{\sigma}_{i, B}=\frac{\sum_{j=1}^{r}(n-j+1) a^{j} D_{i j}+c_{i}}{d_{i}+r+1}=\frac{r \hat{\sigma}_{i}+c_{i}}{d_{i}+r+1} .
$$

- The Bayes estimates (18) is a weighted mean of the mean of the prior $(14)$ and the ML estimate $(29)$; i.e., $\hat{\sigma}_{i, B}=E\left(\sigma_{i}\right) w_{i}+\left(1-w_{i}\right) \hat{\sigma}_{i}$, where $w_{i}=\left(d_{i}-1\right) /\left(d_{i}+r-1\right)$;

- The Bayes estimate (18) is biased, i.e., $\operatorname{Bias}\left(\hat{\sigma}_{i, B}\right)=\left(\sigma_{i}\left(d_{i}-1\right)-c_{i}\right) /\left(d_{i}+r-1\right)$;

- The risk function of the Bayes estimates (18) is

$$
R\left(\hat{\sigma}_{i, B}, \sigma_{i}\right)=\frac{\sigma_{i}^{2}\left(r+\left(1-d_{i}\right)^{2}\right)+2 c_{i}\left(1-d_{i}\right) \sigma_{i}+c_{i}^{2}}{\left(d_{i}+r-1\right)^{2}} ;
$$

- The minimum of $R\left(\hat{\sigma}_{i, B}, \sigma_{i}\right)$, as a function of $\sigma_{i}$, occurs at point $b_{i}\left(d_{i}-1\right) /\left[\left(1-d_{i}\right)^{2}+r\right]$;

- For $r=n$ and $a=1, \hat{\sigma}_{i, n}=\sum_{j=1}^{n} x_{i j} / n$ and $\hat{\sigma}_{i, B}=\left(\sum_{j=1}^{n} x_{i j}+c_{i}\right) /\left(d_{i}+n-1\right)$, which are, respectively, the well-known ML and the Bayes estimates of the exponential parameters on the basis of a random sample of size $n$.

\subsection{Bayesian Test}

Under the null hypothesis $H_{0}: \sigma_{1}=\cdots=\sigma_{s}$, we assume that the common value of $\sigma_{i}(i=1, \cdots, s)$, say $\sigma$, follows the $I G\left(d_{0}, c_{0}\right)$-distribution where $d_{0}$ and $c_{0}$ are known positive hyper parameters. Therefore, the Bayes factor (BF) is derived as (Berger [18])

$$
B F=\frac{c_{0}^{a_{0}} \Gamma\left(s r+d_{0}\right)}{\Gamma\left(d_{0}\right)}\left(\prod_{i=1}^{s} \frac{\left(T_{i}+c_{i}\right)^{d_{i}+1}}{d_{i} c_{i}^{d_{i}}}\right)\left(\sum_{i=1}^{s} T_{i}+c_{0}\right)^{-\left(s r+d_{0}\right)}
$$

Proof. Under the null hypothesis $H_{0}$ in (6), the LF (5) reduces to

$$
L(\sigma ; \boldsymbol{x})=\left(\frac{n !}{(n-r) !}\right)^{s}\left(\prod_{j=1}^{r} a^{j}\right)^{s} \sigma^{-s r} \exp \left\{-\frac{1}{\sigma} \sum_{i=1}^{s} \sum_{j=1}^{r} x_{i j} m_{j}\right\},
$$

and

$$
\begin{aligned}
\pi\left(\boldsymbol{x} \mid H_{0}\right) & =\left(\frac{n !}{(n-r) !}\right)^{s}\left(\prod_{j=1}^{r} a^{j}\right)^{s} \int_{0}^{\infty} \sigma^{-s r} \exp \left\{-\left(\frac{E+c_{0}}{\sigma}\right)\right\} \frac{c_{0}^{d_{0}}}{\Gamma\left(d_{0}\right)} \sigma^{-\left(d_{0}+1\right)} d \sigma \\
& =\left(\frac{n !}{(n-r) !}\right)^{s}\left(\prod_{j=1}^{r} a^{j}\right)^{s} \frac{c_{0}^{d_{0}}}{\Gamma\left(d_{0}\right)} \int_{0}^{\infty} \exp \left\{-\left(\frac{E+c_{0}}{\sigma}\right)\right\} \sigma^{-\left(s r+d_{0}+1\right)} d \sigma
\end{aligned}
$$

where $E=\sum_{i=1}^{s} \sum_{j=1}^{r} x_{i j} m_{j}$. If we use change of variable $x=1 / \sigma$, then

$$
\begin{aligned}
\pi\left(\boldsymbol{x} \mid H_{0}\right) & =\left(\frac{n !}{(n-r) !}\right)^{s}\left(\prod_{j=1}^{r} a^{j}\right)^{s} \frac{c_{0}^{d_{0}}}{\Gamma\left(d_{0}\right)} \int_{0}^{\infty} x^{s r+d_{0}-1} \exp \left\{-\left(E+c_{0}\right) x\right\} d x \\
& =\left(\frac{n !}{(n-r) !}\right)^{s}\left(\prod_{j=1}^{r} a^{j}\right)^{s} \frac{c_{0}^{d_{0}}}{\Gamma\left(d_{0}\right)} \frac{\Gamma\left(s r+d_{0}\right)}{\left(E+c_{0}\right)^{s r+d_{0}}} .
\end{aligned}
$$

Under the alternative hypothesis $H_{1}$ in (6), we have

$$
\begin{aligned}
L\left(\sigma_{1}, \ldots, \sigma_{s} ; \boldsymbol{x}\right) & =\left(\frac{n !}{(n-r) !}\right)^{s}\left(\prod_{j=1}^{r} \alpha_{j}\right)^{s} \prod_{i=1}^{s}\left\{\frac{1}{\sigma_{i}} \exp \left\{-\sum_{j=1}^{r} \frac{x_{i j} m_{j}}{\sigma_{i}}\right\}\right\} \\
& =\left(\frac{n !}{(n-r) !}\right)^{s}\left(\prod_{j=1}^{r} a^{j}\right)^{s} \prod_{i=1}^{s}\left\{\frac{1}{\sigma_{i}} \exp \left\{-\frac{T_{i}}{\sigma_{i}}\right\}\right\},
\end{aligned}
$$


where $T_{i}=\sum_{j=1}^{r} x_{i j} m_{j}$, and

$$
\begin{aligned}
\pi\left(x \mid H_{1}\right)= & \left.\frac{n !}{(n-r) !}\right)^{s}\left(\prod_{j=1}^{r} a^{j}\right)^{s} \prod_{i=1}^{s} \int_{0}^{\infty} \cdots \int_{0}^{\infty} \frac{1}{\sigma_{i}} \exp \left\{-\left(\frac{T_{i}}{\sigma_{i}}\right)\right\} \cdot \frac{c_{i}^{d_{i}}}{\Gamma\left(d_{i}\right)} \cdot \sigma_{i}^{-\left(d_{i}+1\right)} \\
& \times \exp \left\{-\frac{c_{i}}{\sigma_{i}}\right\} d \sigma_{1} \cdots d \sigma_{s} \\
= & \left(\frac{n !}{(n-r) !}\right)^{s}\left(\prod_{j=1}^{r} a^{j}\right)^{s}\left(\prod_{i=1}^{s} \frac{c_{i}^{d_{i}}}{\Gamma\left(d_{i}\right)}\right) \prod_{i=1}^{s} \int_{0}^{\infty} \ldots \int_{0}^{\infty} \sigma_{i}^{-\left(d_{i}+2\right)} \\
& \times \exp \left\{-\left(\frac{T_{i}+c_{i}}{\sigma_{i}}\right)\right\} d \sigma_{1} \cdots d \sigma_{s} .
\end{aligned}
$$

Let $u_{i}=1 / \sigma_{i}$, then

$$
\begin{aligned}
\pi\left(x \mid H_{1}\right)= & \left(\frac{n !}{(n-r) !}\right)^{s}\left(\prod_{j=1}^{r} a^{j}\right)^{s}\left(\prod_{i=1}^{s} \frac{c_{i}^{d_{i}}}{\Gamma\left(d_{i}\right)}\right) \prod_{i=1}^{s} \\
& \times \int_{0}^{\infty} \cdots \int_{0}^{\infty} u_{i}^{d_{i}+1-1} \exp \left\{-\left(T_{i}+c_{i}\right)\right\} d u_{1} \cdots d u_{s} \\
= & \left(\frac{n !}{(n-r) !}\right)^{s}\left(\prod_{j=1}^{r} a^{j}\right)^{s}\left(\prod_{i=1}^{s} \frac{c_{i}^{d_{i}}}{\Gamma\left(d_{i}\right)}\right) \prod_{i=1}^{s}\left(\frac{\Gamma\left(d_{i}+1\right)}{\left(T_{i}+c_{i}\right)^{d_{i}+1}}\right) \\
= & \left(\frac{n !}{(n-r) !}\right)^{s}\left(\prod_{j=1}^{r} a^{j}\right)^{s} \prod_{i=1}^{s}\left(\frac{d_{i} c_{i}^{a_{i}}}{\left(T_{i}+c_{i}\right)^{a d_{i}+1}}\right) .
\end{aligned}
$$

From Equations (22) and (23), the Bayes factor is derived as

$$
B F=\frac{c_{0}^{a_{0}} \Gamma\left(s r+d_{0}\right)}{\Gamma\left(d_{0}\right)}\left(\prod_{i=1}^{s} \frac{\left(T_{i}+c_{i}\right)^{d_{i}+1}}{d_{i} d_{i}}\right)\left(\sum_{i=1}^{s} T_{i}+c_{0}\right)^{-\left(s r+d_{0}\right)} .
$$

Under the " $0-K_{i}$ " loss function (Berger [18]), the Bayes test rejects the null hypothesis $H_{0}$ in (6) if

$$
B F<\left(\frac{K_{0}}{k_{1}}\right)\left(\frac{\pi_{1}}{\pi_{0}}\right)
$$

where $\pi_{i}$ and $K_{i}$, for $i=1,2$, are prior probability for the hypothesis $H_{i}$ and the loss of the accepting $H_{i}$ when $H_{j}(j \neq i)$ is correct, respectively.

\subsection{HPD Credible Sets}

In this section, the problem of finding highest posterior density (HPD) credible set is considered. To do this, from Equation (16), we see that

$$
R_{i}=2\left(\frac{\sum_{j=1}^{r}(n-j+1) a^{j} D_{i j}+c_{i}}{\sigma_{i}}\right) \mid \mathbf{x} \sim \chi_{2\left(d_{i}+r\right)}^{2} .
$$

Therefore, an equi-tailed credible set at level $\gamma$ for $\sigma_{i}(i=1, \cdots, s)$ is obtained as

$$
C_{i}(\gamma)=\left(\frac{\sum_{j=1}^{r}(n-j+1) a^{j} D_{i j}+c_{i}}{\chi_{2\left(d_{i}+r\right),(1+\gamma) / 2}^{2}}, \frac{\sum_{j=1}^{r}(n-j+1) a^{j} D_{i j}+c_{i}}{\chi_{2\left(d_{i}+r\right),(1-\gamma) / 2}^{2}}\right) .
$$

The HPD credible set for $\sigma_{i}(i=1, \cdots, s)$ is

$$
\left\{\sigma_{i}: \sigma_{i}^{-\left(d_{i}+r\right)-1} \exp \left\{-\frac{\sum_{j=1}^{r}(n-j+1) a^{j} D_{i j}+c_{i}}{\sigma_{i}}\right\}>k\right\},
$$


where $k$ is a constant determined by the condition on the level of the credible set, i.e.,

$$
P\left(\sigma_{i}^{-\left(d_{i}+r\right)-1} \exp \left\{-\left(\frac{\sum_{j=1}^{r}(n-j+1) a^{j} D_{i j}+c_{i}}{\sigma_{i}}\right)\right\}>k \mid \mathrm{x}\right)=\gamma .
$$

Hence, the HPD credible set for $\sigma_{i}(i=1, \cdots, s)$ in Equation (26) is an interval, say $\left(L_{i}, U_{i}\right)$, where $L_{i}$ and $U_{i}$ are derived from the following non-linear equations:

$$
\left\{\begin{array}{l}
\left(\frac{U_{i}}{L_{i}}\right)^{d_{i}+r+1}=\exp \left\{\left(\frac{U_{i}-L_{i}}{U_{i} L_{i}}\right) \Psi_{i}(\mathbf{x}, a)\right\} \\
F_{\chi_{2\left(d_{i}+r\right)}^{2}}\left(\frac{2 \Psi_{i}(\mathbf{x}, a)}{U_{i}}\right)-F_{\chi_{2\left(d_{i}+r\right)}^{2}}\left(\frac{2 \Psi_{i}(\mathbf{x}, a)}{L_{i}}\right)=\gamma_{i}\left(\frac{\Gamma\left(d_{i}\right)}{\Gamma\left(d_{i}+r\right)} c_{i}^{-d_{i}} a^{\frac{r(r+1)}{2}}\left(4 \Psi_{i}(\mathbf{x}, a)\right)^{d_{i}+r}\right)
\end{array}\right.
$$

where $\Psi_{i}(\mathbf{x}, a)=\sum_{j=1}^{r}(n-j+1) a^{j} D_{i j}+c_{i}$, and $F_{\chi_{\nu}}(t)$ stands for the CDF of the $\chi_{\nu}$-distribution. For the proof see Appendix B. In practice, one may use numerical methods for solving the above equations.

\section{CONCLUSIONS}

In this paper, based on independent SOSs coming from non-homogeneous exponential populations, the ML and the Bayes estimates of parameters were obtained on the basis of multiple SOS samples. The GLRT and Bayesian test were also derived for testing homogeneity of the exponential baseline populations. In Bayesian inference, when the parameter $a$ is unknown, the mentioned problems are complicated and works in this direction are under investigation and we hope to report findings in future. The results of this paper may be extended in some directions. For example, the Bayes estimates may by obtained under some asymmetric loss functions such as linear-exponential loss function.

\section{REFERENCES}

1. R.E. Barlow, F. Proschan, Statistical Theory of Reliability and Life Testing: Probability Models, second ed., Springer, New York, USA, 1981.

2. H.A. David, H.N. Nagaraja, Order Statistics, John Wiley \& Sons, Inc., Hoboken, New Jersey, USA, 2003.

3. E. Cramer, U. Kamps, Ann. Inst. Stat. Math. 48 (1996), 535-549.

4. U. Kamps, A Concept of Generalized Order Statistics, Teubner, Wiesbaden, Germany, 1995.

5. U. Kamps, J. Stat. Plan. Inference. 48 (1995), 1-23.

6. E. Cramer, U. Kamps, Ann. Inst. Stat. Math. 53 (2001), 307-324.

7. N. Balakrishnan, U. Kamps, M. Kateri, Ann. Inst. Stat. Math. 64 (2012), 302-318.

8. E. Beutner, U. Kamps, J. Stat. Plan. Inference. 139 (2009), 2963-2969.

9. M. Hashempour, Classical, Bayesian and Evidential Inferences Based on Sequential Order Statistics, PhD Thesis in Mathematical Statistics, Department of Statistics, Ferdowsi University of Mashhad, Mashhad, Iran, 2017.

10. S. Bedbur, J. Stat. Plan. Inference. 140 (2010), 2520-2530.

11. M. Hashempour, M. Doostparast, Commun. Stat. Theor. Methods. 46 (2016), 8086-8100.

12. E. Cramer, U. Kamps, in: N. Balakrishnan, E. Rao (Eds.), Handbook of Statistics, Advances in Reliability, Elsevier, New York, USA, vol. 20, chap. 12, 2001, pp. 301-372.

13. N. Schenk, Point Estimation with Sequential Order Statistics Based on Exponential Distributions, PhD Thesis, University of Oldenburg, Oldenburg, Germany, 2001.

14. E.L. Lehmann, J. Romano, Testing Statistical Hypothesis, third ed., Springer, New York, USA, 2005.

15. M.M. Mohie El-Din, M.M. Amein, M.S. Moham, J. Adv. Res. Appl. Math. 5 (2013), 80-96.

16. N. Schenk, M. Burkschat, E. Cramer, U. Kamps, J. Stat. Plan. Inference. 141 (2011), 1575-1587.

17. A.R. Shafay, N. Balakrishnan, K.S. Sultan, J. Stat. Comput. Simul. 84 (2014), 526-544.

18. J. Berger, Statistical Decision Theory and Baysian Analysis, second ed., Springer, New York, USA, 1985.

19. E.L. Lehmann, G. Casella, Theory of Point Estimation, second ed., Springer, New York, USA, 1998. 


\section{APPENDIX A.}

Suppose that the parameter $a$ in Equation (5) is known. Under the null hypothesis $H_{0}$ in (6) (Cramer and Kamps [12]), the unique ML estimate of the common mean of the $s$ exponential populations, say $\sigma_{0}$, is

$$
\hat{\sigma}_{0}=\frac{1}{r s} \sum_{i=1}^{s} \sum_{j=1}^{r}(n-j+1) a^{j} D_{i j}=\frac{1}{r s} \sum_{i=1}^{s} \sum_{j=1}^{r} x_{i j} m_{j},
$$

where $D_{i j}=x_{i j}-x_{i, j-1}$, for $j=1, \cdots, r$. Here $x_{i 0}:=0$ for $i=1, \cdots, s$. When the baseline exponential populations are heterogeneous, from Equation (28), the unique ML estimate of $\sigma_{i}(i=1, \cdots, s)$ is derived as

$$
\hat{\sigma}_{i}=\frac{1}{r} \sum_{j=1}^{r}(n-j+1) a^{j} D_{i j}=\frac{1}{r} \sum_{j=1}^{r} x_{i j} m_{j} .
$$

Corollary 1. Under the PTCPHR with the one-parameter exponential baseline CDF,

$$
T_{i}=\sum_{j=1}^{r}(n-j+1) a^{j} D_{i j} \sim \Gamma\left(r, \sigma_{i}\right), \quad i=1, \cdots, s,
$$

where $\Gamma(a, b)$ calls for the gamma distribution with shape and scale parameters a and b, respectively. Notice that $\sum_{j=1}^{r} x_{i j} m_{j}=\sum_{j=1}^{r}(n-j+1)$ $a^{j} D_{i j}$, for $i=1, \cdots, s$.

Now consider the problem of hypotheses testing (6). The generalized likelihood ratio test (GLRT) statistic for testing the problem (6) is

$$
\Lambda_{1}=\prod_{i=1}^{s}\left(\frac{\hat{\sigma}_{i}}{\hat{\sigma}_{0}}\right)^{r} \exp \left\{\sum_{i=1}^{s} \sum_{j=1}^{r}\left(\frac{1}{\hat{\sigma}_{i}}-\frac{1}{\hat{\sigma}_{0}}\right) m_{j} x_{i j}\right\},
$$

where $\Omega=\left\{\left(\sigma_{1}, \cdots, \sigma_{s}\right): \sigma_{i}>0, i=1, \cdots, s\right\}=: \mathbb{R}^{+s}$ is the whole parameter space and $\Omega_{0}=\left\{\left(\sigma_{1}, \ldots, \sigma_{s}\right): \sigma_{1}=\cdots=\sigma_{s}\right\}$ denotes the parameter space under the null hypothesis $H_{0}$. After some algebraic manipulations, the logarithm of the GLRT statistic $\Lambda_{1}$ given by Equation (31) reduces to

$$
\log \Lambda_{1}=r \sum_{i=1}^{s} \log \left(\frac{s T_{i}}{\sum_{j=1}^{s} T_{j}}\right)
$$

where $T_{i}$ is defined by Equation (30) and "log" stands for the natural logarithm. Under the null hypothesis $H_{0}$ in (6) and the usual regularity conditions (see Lehmann and Cassella [19]), $-2 \log \Lambda_{1}$ has asymptotically the chi-square distribution with 1 degree of freedom. Thus, for large $n$, the rejection region of the GLR test of size $\gamma$ is

$$
-2 \log \Lambda_{1}>\chi_{1,1-\gamma}^{2}
$$

where $\chi_{v, \gamma}^{2}$ is the $\gamma$-th percentile of the chi-square distribution with $v$ degrees of freedom. Also, the actual level of the GLRT may be obtained by conducting a Monte Carlo simulation study for given $a$ and $\sigma$.

The observed Fisher Information, denoted by $i\left(\hat{\sigma}_{1}, \cdots, \hat{\sigma}_{s}\right)$, on the basis of available SOSs data is equal to minus of the Hessian matrix evaluated at the MLEs of the parameters, i.e.,

$$
i\left(\hat{\sigma}_{1}, \cdots, \hat{\sigma}_{s}\right)=\left.\left[\left[\left(-\partial^{2} \log (L) / \partial \sigma_{i} \partial \sigma_{j}\right)_{1 \leq i, j \leq s}\right]\right]\right|_{\sigma_{1}=\hat{\sigma}_{1}, \cdots, \sigma_{s}=\hat{\sigma}_{s}}
$$

It is well known that the unique MLEs have asymptotically the multivariate normal distribution with mean vector $\left(\sigma_{1}, \cdots, \sigma_{s}\right)$ and the variance-covariance matrix $\left[i\left(\hat{\sigma}_{1}, \cdots, \hat{\sigma}_{s}\right)\right]^{-1}$. Here, the observed Fisher Information for $\sigma_{i}$ is derived as

$$
i\left(\hat{\sigma}_{1}, \cdots, \hat{\sigma}_{s}\right)=\operatorname{diag}\left[\left[\frac{r}{\widehat{\sigma}_{i j}}\right]\right]
$$

Notice that, by Equation (30), one can see that $2 r\left(\hat{\sigma}_{i} / \sigma_{i}\right) \sim \chi_{2 r}^{2}$. Hence, an approximate equi-tailed confidence interval for $\sigma_{i}$ is

$$
\left(\hat{\sigma}_{i}-z_{\gamma / 2} \sqrt{\frac{\hat{\sigma}_{i}^{2}}{r}}, \hat{\sigma}_{i}+z_{\gamma / 2} \sqrt{\frac{\hat{\sigma}_{i}^{2}}{r}}\right),
$$


where $z_{\gamma}$ stands for the $\gamma$-percentile of the standard normal distribution. Therefore, an equal-tail exact confidence interval at level $100 \gamma \%$ for $\sigma_{i}(i=1, \cdots, s)$ is

$$
\left(\frac{2 r \hat{\sigma}_{i}}{\chi_{2 r,(1+\gamma) / 2}^{2}}, \frac{2 r \hat{\sigma}_{i}}{\chi_{2 r,(1-\gamma) / 2}^{2}}\right)
$$

where $\chi_{\nu, p}^{2}$ calls for the $p$-th percentile of the $\chi_{\nu}^{2}$-distribution.

\section{APPENDIX B.}

By definition, we have

$$
\int_{L_{i}}^{U_{i}} \prod_{j=1}^{r} a^{j} \frac{c_{i}^{d_{i}}}{\Gamma\left(d_{i}\right)} \sigma_{i}^{-\left(d_{i}+r\right)-1} \exp \left\{-\left(\frac{\sum_{j=1}^{r} x_{i j} m_{j}+c_{i}}{\sigma_{i}}\right)\right\} d \sigma_{i}=\gamma_{i}
$$

Let $t_{i}=2 \Psi(\mathbf{x}, a) / \sigma_{i}$, then

$$
\begin{aligned}
\gamma_{i} & =\int_{\frac{2 \Psi(\mathbf{x}, a)}{U_{i}}}^{\frac{2 \Psi(\mathbf{x}, a)}{L_{i}}} \prod_{j=1}^{r} a^{j} \frac{c_{i}^{d_{i}}}{\Gamma\left(d_{i}\right)}\left(\frac{2 \Psi(\mathbf{x}, a)}{t_{i}}\right)^{-\left(d_{i}+r\right)-1} \exp \left\{-\frac{t_{i}}{2}\right\}\left(-\frac{2 \Psi(\mathbf{x}, a)}{t_{i}^{2}}\right) d t_{i} \\
& =\prod_{j=1}^{r} a^{j} \frac{c_{i}^{d_{i}}}{\Gamma\left(d_{i}\right)} \int_{\frac{2 \Psi(\mathbf{x}, a)}{U_{i}}}^{\frac{2 \Psi(\mathbf{x}, a)}{L_{i}}}(2 \Psi(\mathbf{x}, a))^{-\left(d_{i}+r\right)-1}\left(t_{i}\right)^{d_{i}+r+1} \exp \left\{-\frac{t_{i}}{2}\right\}\left(-\frac{2 \Psi(\mathbf{x}, a)}{t_{i}^{2}}\right) d t_{i} \\
& =-a^{\frac{r(r+1)}{2}} \frac{c_{i}^{d_{i}}}{\Gamma\left(d_{i}\right)}(2 \Psi(\mathbf{x}, a))^{-\left(d_{i}+r\right)} \int_{\frac{2 \Psi(\mathbf{x}, a)}{U_{i}}}^{\frac{2 \Psi(\mathbf{x}, a)}{L_{i}}} t_{i}^{\left(d_{i}+r\right)-1} \exp \left\{-\frac{t_{i}}{2}\right\} d t_{i} \\
& =-a \frac{r(r+1)}{2} \frac{a_{\left(d_{i}+r\right)}}{\Gamma\left(d_{i}\right)} d_{i}^{c_{i}}\left(\frac{2}{\Psi(\mathbf{x}, a)}\right)^{\frac{\left(d_{i}+r\right)}{\left.L^{2}, a\right)}} \int_{2}^{2 \frac{\Psi(\mathbf{x}, a)}{U_{i}}} \frac{t_{i}^{\left(d_{i}+r\right)-1}}{\Gamma\left(d_{i}+r\right) 2^{d_{i}+r}} \exp \left\{-\frac{t_{i}}{2}\right\} d t_{i} \\
& =-\frac{\Gamma\left(d_{i}+r\right)}{\Gamma\left(d_{i}\right)} c_{i}^{d_{i}}\left(\frac{2}{\Psi(\mathbf{x}, a)}\right)^{\left(d_{i}+r\right)} a^{\frac{r(r+1)}{2}}\left(F_{\chi_{2}\left(r+d_{i}\right)}\left(\frac{2 \Psi(\mathbf{x}, a)}{L_{i}}\right)-F_{\chi_{2}\left(r+d_{i}\right)}\left(\frac{2 \Psi(\mathbf{x}, a)}{U_{i}}\right)\right) .
\end{aligned}
$$

After some algebraic calculations, $\gamma_{i}$ is simplified to

$$
a^{\frac{r(r+1)}{2}}\left(\frac{\Gamma\left(d_{i}+r\right)}{\Gamma\left(d_{i}\right)} c_{i}^{d_{i}}\left(4 \Psi_{i}(\mathbf{x}, a)\right)^{d_{i}+r}\right)\left(F_{\chi_{2\left(d_{i}+r\right)}^{2}}\left(\frac{2 \Psi(\mathbf{x}, a)}{U_{i}}\right)-F_{\chi_{2\left(d_{i}+r\right)}^{2}}\left(\frac{2 \Psi(\mathbf{x}, a)}{L_{i}}\right)\right)=\gamma_{i},
$$

and then

$$
F_{\chi_{2\left(d_{i}+r\right)}^{2}}\left(\frac{2 \Psi_{i}(\mathbf{x}, a)}{U_{i}}\right)-F_{\chi_{2\left(d_{i}+r\right)}^{2}}\left(\frac{2 \Psi_{i}(\mathbf{x}, a)}{L_{i}}\right)=\gamma_{i}\left(\frac{\Gamma\left(d_{i}\right)}{\Gamma\left(d_{i}+r\right)} c_{i}^{-d_{i}} a^{\frac{r(r+1)}{2}}\left(4 \Psi_{i}(\mathbf{x}, a)\right)^{d_{i}+r}\right) .
$$

Since $\pi\left(L_{i} \mid x\right)=\pi\left(U_{i} \mid x\right)$, then

$$
\begin{aligned}
L_{i}^{-\left(d_{i}+r\right)-1} \exp \left\{-\left(\frac{\Psi_{i}(\mathbf{x}, a)}{L_{i}}\right)\right\} & =U_{i}^{-\left(d_{i}+r\right)-1} \\
\exp \left\{-\left(\frac{\Psi_{i}(\mathbf{x}, a)}{U_{i}}\right)\right\} \text { and }\left(\frac{U_{i}}{L_{i}}\right)^{d_{i}+r+1} & =\exp \left\{\frac{\Psi_{i}(\mathbf{x}, a)}{L_{i}}-\frac{\Psi_{i}(\mathbf{x}, a)}{U_{i}}\right\} \\
& =\exp \left\{\frac{U_{i}-L_{i}}{U_{i} L_{i}}\left(\Psi_{i}(\mathbf{x}, a)\right) .\right\} .
\end{aligned}
$$

Finally,

$$
\left\{\begin{array}{l}
\left(\frac{U_{i}}{L_{i}}\right)^{d_{i}+r+1}=\exp \left\{\left(\frac{U_{i}-L_{i}}{U_{i} L_{i}}\right) \Psi_{i}(\mathbf{x}, a)\right\} \\
F_{\chi_{2\left(d_{i}+r\right)}^{2}}\left(\frac{2 \Psi_{i}(\mathbf{x}, a)}{U_{i}}\right)-F_{\chi_{2\left(d_{i}+r\right)}^{2}}\left(\frac{2 \Psi_{i}(\mathbf{x}, a)}{L_{i}}\right)=\gamma_{i}\left(\frac{\Gamma\left(d_{i}\right)}{\Gamma\left(d_{i}+r\right)} c_{i}^{-d_{i}} a^{\frac{r(r+1)}{2}}\left(4 \Psi_{i}(\mathbf{x}, a)\right)^{d_{i}+r}\right) .
\end{array}\right.
$$

